\title{
Interventions to reduce occupational stress and burn out within neonatal intensive care units: a systematic review
}

\author{
Ilia Bresesti (1) , 1,2 Laura Folgori, ${ }^{1,3}$ Paola De Bartolo ${ }^{2,4}$
}

\begin{abstract}
- Additional material is published online only. To view please visit the journal online (http://dx.doi.org/10.1136/ oemed-2019-106256).

${ }^{1}$ Department of Pediatrics, Luigi Sacco University Hospital, Milano, Italy

${ }^{2}$ Department of Human Science, Guglielmo Marconi University,

Roma, Italy

${ }^{3}$ Pediatric Infectious Diseases Research Group, Institute for Infection and Immunity, St George's University of London, London, UK

${ }^{4}$ Laboratory of Experimental Neurofisiology, IRCCS

Fondazione Santa Lucia, Roma, Italy
\end{abstract}

\section{Correspondence to}

Dr llia Bresesti, Department of Pediatrics, Luigi Sacco University Hospital, Milano 20157, Italy; ilia_bresesti@hotmail.it

Received 11 October 2019 Revised 22 January 2020 Accepted 18 February 2020

\section{ABSTRACT}

Occupational stress is an emerging problem among physician and nurses, and those working in intensive care settings are particularly exposed to the risk of developing burnout. To verify what types of interventions to manage occupational stress and burn out within neonatal intensive care units (NICUs) have been introduced so far and to verify their efficacy among caregivers. PsycINFO (PsycINFO 1967-July week 3 2019) Embase (Embase 1996-2019 week 29) e Medline (Ovid MEDLINE(R) without revisions 1996-July week 2 2019) were systematically searched combining MeSH and free text terms for "burn out" AND "healthcare provider" AND "NICU". Inclusion criteria were interventions directed to healthcare providers settled in NICUs. Only English language papers were included. Six articles were included in the final analysis. All the studies reported an overall efficacy of the interventions in reducing work-related stress, both when individual focused and organisation directed. The analysis revealed low quality of the studies and high heterogeneity in terms of study design, included populations, interventions and their evaluation assessment. There is currently very limited evidence regarding the management of occupational stress and burn out within NICUs. The quality of available studies was suboptimal. The peculiarities of the NICUs should be considered when developing strategies for occupational stress management. Training self-awareness of workers regarding their reactions to the NICU environment, also from the pre-employment stage, could be an additional approach to prevent and manage stress.

\section{INTRODUCTION}

The term burn out refers to a work-related syndrome characterised by feelings of emotional exhaustion, depersonalisation and a sense of reduced personal accomplishment in response to prolonged exposure to occupational stress. ${ }^{1}$ Workers in service professions such as healthcare are more prone to this syndrome. ${ }^{1}$

Although recent evidence has reported an overall decrease in the prevalence of burn out among US physicians, they remain at increased risk compared with workers in other fields. ${ }^{2}$ Consequences of this syndrome involve not only personal life quality of medical staff, ${ }^{2}$ but it also may have an impact on productivity, quality of patient care and lower patient satisfaction. ${ }^{3-7}$ Furthermore, burn out may exacerbate staffing shortages. ${ }^{8}$ All healthcare providers working at the front line of care are at higher risk for burn out development, ${ }^{12}$ and up to $50 \%$ of those dealing with the most vulnerable
Key messages

What is already known about this subject?

- Burn out in is an emerging problem in medical settings and may have a relevant impact on both well-being of workers and efficiency of the healthcare organisation.

- Healthcare providers in neonatal intensive care units (NICUs) are particularly exposed to occupational stress.

- There is still high uncertainty about how to manage this problem in medical setting.

What are the new findings?

- A very limited number of studies have explored the management of occupational stress and burn out in workers of NICUs. These studies pointed out high heterogeneity and overall poor quality which precluded from significant conclusions.

How might this impact on policy or clinical practice in the foreseeable future?

- The peculiarities of the NICU should be considered when developing strategies for occupational stress management. Approaching workers also from the pre-employment stage might be an additional intervention to prevent stress. NICU workers might benefit from a tailored approach, although there is urgent need for further research in this area.

infants in the neonatal intensive care unit (NICU) suffer from this syndrome. ${ }^{9-11}$

Other than high workload and frequently understaffed team, the NICU has some peculiarities which may explain the increase of occupational stress in this setting. Neonatal care has witnessed substantial and rapid changes in the last decades, and continuous advances in technology expose NICU providers to frequent changes in clinical practice. Moreover, these improvements have led to the care of extreme prematurity, which is accompanied by even more high expectation from parents. Hospital stay has become longer and parental presence in the NICU is so actively promoted that commitment to reduce mother and father stress is nowadays part of the routine care. This approach establishes a deep emotional involvement between parents and healthcare providers. Furthermore, the higher level of care, the more nurses and neonatologist must confront ethical dilemmas related to end-of-life 

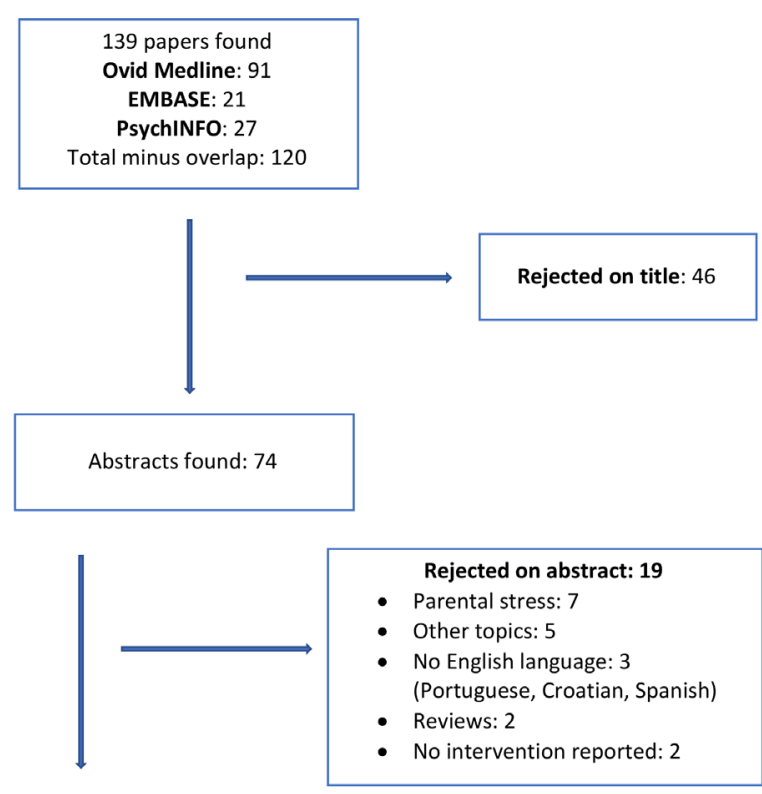

Full text found: 55

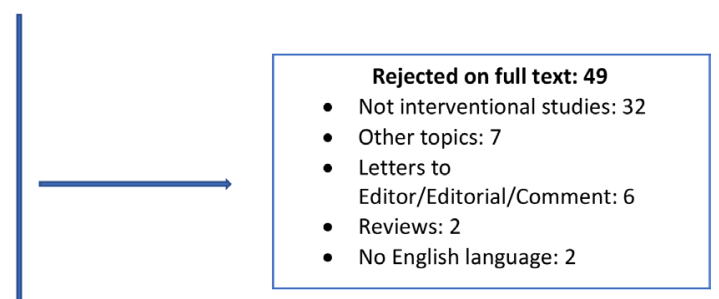

Accepted studies: 6

Figure 1 Flowchart and study selection.

care and innovative medicine, which in turn promotes moral distress development. ${ }^{12}$

Given these premises, the introduction of interventions to manage occupational stress and prevent burn out within NICUs is necessary. There is a lack of evidence regarding the approaches to professional stress reduction which are most suited for use in NICUs. The aim of this review was to investigate what types of interventions have been introduced within NICUs so far and to verify their efficacy among caregivers.

\section{METHODS}

\section{Search strategy and selection criteria}

A systematic review of the literature was conducted to investigate interventions for managing occupational stress and/or burnout in healthcare providers working in NICUs.

PsycINFO (PsycINFO 1967-July week 3 2019), Embase (Embase 1996-2019 week 29) e Medline (Ovid MEDLINE(R) without revisions 1996-July week 2 2019) electronic databases were systematically searched on 23 July 2019 using a strategy combining MeSH and free text terms for "burn out" AND "healthcare provider" AND "NICU". No time limits were applied. The full search strategy is available as online supplementary material.

Reference lists of papers which underwent full-text analysis were scanned for additional studies if eligibility criteria were met. Studies (1) reporting interventions against work-related stress and/or burnout; (2) involving healthcare providers; (3) settled in NICU were included. Both interventions focused on individuals (eg, cognitive behavioural therapies, mindfulness-based stress reduction techniques, educational programmes for improving communication skills) and organisation-directed (eg, changes in the resources, the working environment and/or organisation of teamwork) were included. No restrictions were made regarding the type of intervention. Studies involving students selectively were excluded. Only studies published in English were included.

This study did not receive any direct funding. The study complies with the Preferred Reporting Items for Systematic Reviews and Meta-Analyses guideline. ${ }^{13}$

\section{Data extraction}

For each included study, two authors (IB and LF) independently extracted the following features according to prespecified criteria: study characteristics (design, year, country), participants (sample size, role in NICU), intervention (content, duration, efficacy assessment) and outcomes (scores in occupational stress and burnout). In case of disagreements, these were resolved in discussion with a third author (PDB).

\section{Data analysis}

Occupational stress and burn out reduction were considered as primary outcomes and no restrictions were applied in regard to the measuring methods (eg, self-evaluation, use of validates tools such as stress-scales, biological markers of stress). Main characteristics of the studies such as design, population, duration, secondary outcomes and follow-up were analysed, where available.

\section{Quality assessment}

The quality of the selected articles was assessed using the integrated quality criteria for systematic review of multiple study design' (ICROMS) tool. ${ }^{14}$ This instrument is sufficiently flexible to be applicable to a wide range of study designs and outcome measures. The 'decision matrix' is made of two separate parts. For each study design, there are different mandatory criteria to be met. In addition, a quality score is calculated addressing several questions, which are specific for each study type. Once both components are met, the study is of sufficient value to be included in systematic reviews.

\section{RESULTS}

Our search identified 139 papers. A total of 120 studies were reviewed on the basis of title and abstract after removal of duplicates. The full text of 55 papers was assessed. Two papers were unavailable for full-text review after the abstract revision and could not be assessed. ${ }^{15} 16$ Six articles fulfilled the inclusion and exclusion criteria and were included in the final analysis ${ }^{17-22}$ (figure 1).

\section{Characteristics of studies and population}

Characteristics of the included studies are outlined in table 1.

Four out of six studies were conducted in the USA, ${ }^{17-20}$ one in the $\mathrm{UK}^{21}$ and one in Iran. ${ }^{22}$ Only four studies reported the sample size, for a total of 275 healthcare providers involved. The 
万人.

离

告

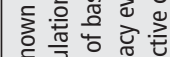

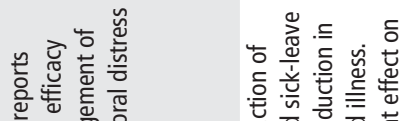

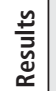

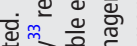

은

훈

管

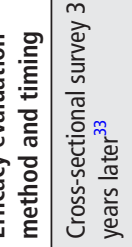

흘 $\overline{\bar{\sigma}}$ व

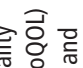

훌을 을

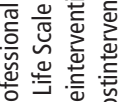

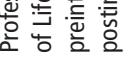

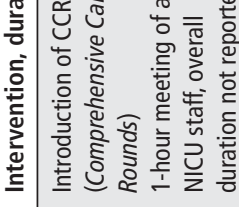

氙

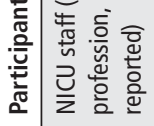

离

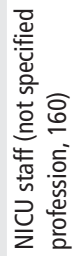

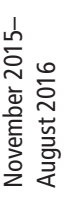

空

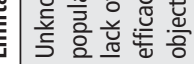

IIn⿴囗十)

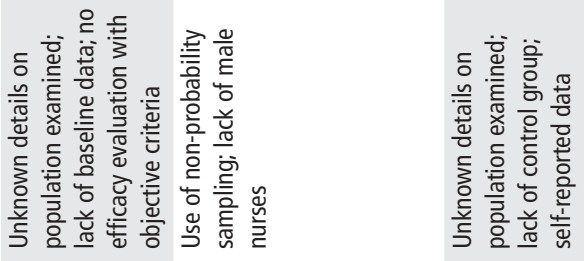

密言䓂言

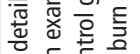

釷

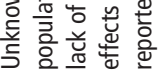

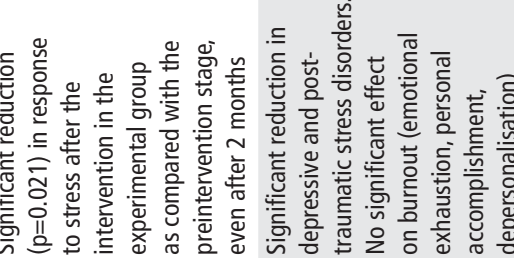

突蜜

宫员产

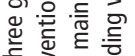

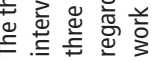

강

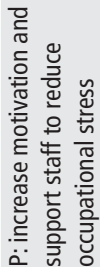

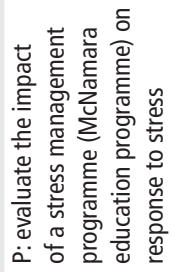

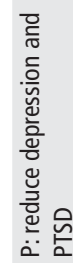

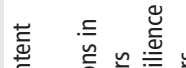

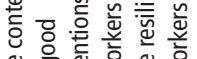

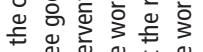

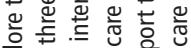

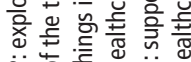

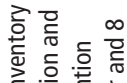

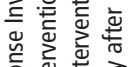

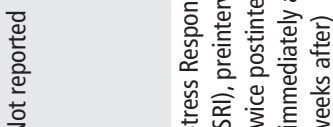

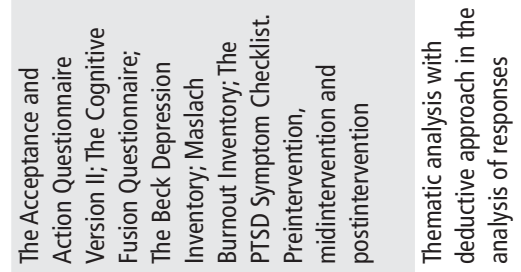

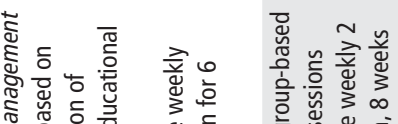

은

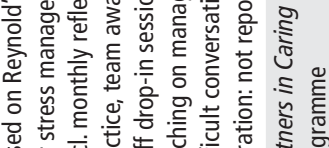

WIIII

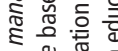

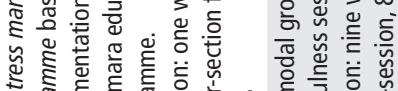

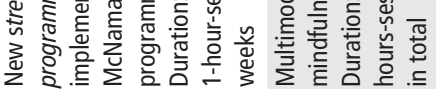

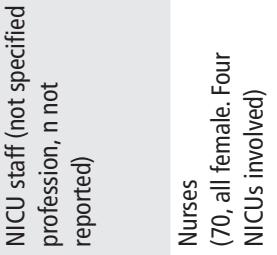

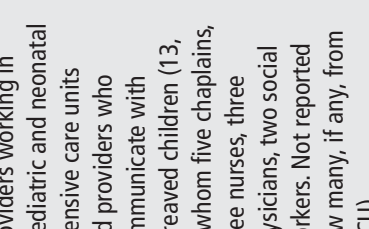

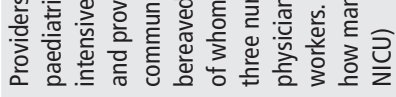

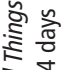

응

苋

崩 总

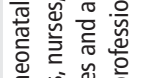

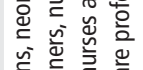

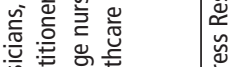

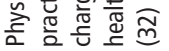

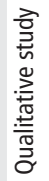

$\Sigma$

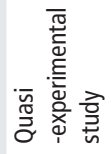

흘

홀

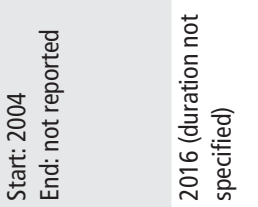

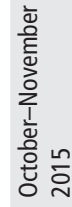

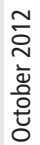

药 跒

$\overleftarrow{s}$

荘

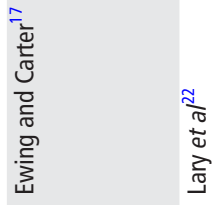

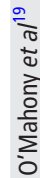


majority of studies were performed within the last 10 years, ${ }^{19-22}$ and two dates back to early 2000s. ${ }^{17} 18$

Four of the selected studies reported data of a single NICU, ${ }^{17} 182021$ one study involved multiple NICUs ${ }^{22}$ whereas one study did not report this data. ${ }^{19}$

Participants' gender was specified in one study, in which all the subjects included were female. ${ }^{22}$ Two papers reported the mean age of participants. ${ }^{19} 22$ The degree of experience was specified in one study as an inclusion criterion. ${ }^{22}$ None of the studies specifically targeted providers with certain severity levels of occupational stress or burnout.

In three studies, although settled in NICU, participants' profession was not specified. ${ }^{171821}$ One study recruited participants also among NICU workers, but the paper did not specify how many, if any, were included in the study. ${ }^{20}$

Stress and burn out assessment tool varied considerably between studies. One study used the Maslac Burnout Inventory (MBI), ${ }^{19}$ one used the Professional Quality of Life Scale, ${ }^{21}$ one used the Stress Response Inventory ${ }^{22}$ and one study utilised multiple tools (the Acceptance and Action Questionnaire Version II, the Cognitive Fusion Questionnaire, the Beck Depression Inventory, the MBI, the Post Traumatic Stress Disorder (PTSD) Symptom Checklist). ${ }^{19}$ Finally, one qualitative study used thematic analysis with deductive approaches. ${ }^{20}$ Two papers did not report the stress evaluation method. ${ }^{17} 18$

\section{Characteristics of interventions}

High heterogeneity was found in terms of intervention characteristics (content, duration, efficacy assessment) between the selected studies. Three studies described individual-based interventions, including mindfulness-based technique, educational interventions to improve communication and stress coping skills, positive emotions empowerment. ${ }^{19} 2022$ Three studies described organisation-directed interventions, ${ }^{17} 1821$ including structural changes in the unit management and providers' interventions such as routine staff meetings, discussion groups on ethical issue and motivational activities. The duration of the interventions, where specified, ranged from 2 to 8 weeks. Only one study performed a short-term follow-up ( 8 weeks after the intervention cessation). ${ }^{22}$

\section{Quality assessment}

Results are outlined in table 2.

Given the paucity of included studies, the quality was not considered as an exclusion criterion.

Two studies had not sufficient criteria for quality assessment, ${ }^{17} 18$ three studies showed suboptimal quality because they

Table 2 Score attributed to selected articles according to integrated quality criteria for systematic review of multiple study design tool

\begin{tabular}{|c|c|c|c|c|}
\hline Reference & $\begin{array}{l}\text { Study } \\
\text { design }\end{array}$ & $\begin{array}{l}\text { Minimum } \\
\text { score } \\
\text { required }\end{array}$ & $\begin{array}{l}\text { Mandatory } \\
\text { criteria met }\end{array}$ & $\begin{array}{l}\text { Article } \\
\text { score }\end{array}$ \\
\hline Boos et $a l^{18}$ & NA & NA & NA & NA \\
\hline$D^{\prime}$ Urso et $a l^{21}$ & NCBA & 22 & No & 12 \\
\hline Ewing and Carter ${ }^{17}$ & NA & NA & NA & NA \\
\hline Lary et $a l^{22}$ & CBA & 18 & Yes & 25 \\
\hline O'Mahony et all ${ }^{19}$ & NCBA & 22 & No & 19 \\
\hline $\begin{array}{l}\text { Rippstein-Leuenberger } \\
\text { et } \mathrm{al}^{20}\end{array}$ & QUAL & 16 & No & 14 \\
\hline
\end{tabular}

did not meet the minimum score or mandatory criteria for study quality. ${ }^{19-21}$ One paper fulfilled the criteria for study quality according to ICROMS tool. ${ }^{22}$

\section{DISCUSSION}

The main aim of this review was to summarise the current evidence regarding occupational stress and burn out management among caregivers working in NICUs. The reviewed papers differed significantly in terms of population studied, intervention types, study design and methods for efficacy evaluation. This considerable heterogeneity, together with the scarcity of quality among reviewed studies, precluded us from drawing broad conclusions. Of the six papers analysed, there was no randomised controlled trial, and two studies reported interventions for stress management in a descriptive way, without a clear report of methods and results, then making the study design definition not possible. ${ }^{17} 18$ Our results substantiate that both individual-directed and organisational-focused interventions could reduce occupational stress. Where specified, studies reported that the interventions were overall effective on at least one expressions of stress, regardless of the intervention type. Effects on burnout as a specific outcome remains controversial.

Other systematic reviews have examined the effects of interventions on work-related stress in healthcare professionals. ${ }^{23-27}$ West and colleagues conducted a review on physician burnout, and concluded for an overall efficacy of the interventions, both individual and organisational directed. ${ }^{24}$ Panagioti et al partly confirmed these findings, but their meta-analysis highlighted how organisational improvements might boost physiciandirected intervention, which may be of small benefits if applied alone. ${ }^{26}$ Clough and colleagues underlined the potential benefits of psychosocial and behavioural interventions. ${ }^{25}$ In this regard, the use of mindfulness has been previously described in healthcare providers, showing promising results. ${ }^{28-32}$ All these studies, however, pointed out the poor quality of the studies conducted so far and underlined the urgent need for higher quality research in this field.

To date, there is no clear evidence about which intervention are the most effective for specific populations of workers. To our knowledge, this is the first comprehensive review about occupational stress and burn out management settled in NICU.

This study has some limitations. The primary limits are the small number of studies included and the poor overall quality. The high heterogeneity among the included studies precluded meaningful meta-analysis. In regard to study populations, given the lack of reported information about clinical experience, age and other confounding factors in the majority of the reviewed studies, caution is advised when interpreting the results. In addition, NICU characteristics were not specified (number of beds, average daily admissions, mean length of stay, mortality rate, etc) thus limiting the generalisability of the results. The lack of follow-up data influenced the efficacy evaluation of the interventions.

\section{CONCLUSION}

Although burn out is proven to be common among caregivers dealing with neonatal population and working in intensive care, studies approaching this phenomenon and its management in a systematic way seem to be far from optimal. The high heterogeneity of studies does not allow recommendations to be made about burn out management in NICU. The peculiarities of the NICU environment should be considered when developing strategies for occupational stress management. Workers of this 
specific setting might benefit from a tailored approach. The exposure to constant moral distress, the emotional exhaustion deriving from caring for critically ill neonates, the need for daily controversial ethical decision and their impacts on a developing family may deeply interplay with the individual coping strategies and also with psychological traits. Cognitive rigidity, scarce stress tolerance, poor or extreme empathic attitude, relational and communicative difficulty are personality's features which can be exacerbated in specific occupational fields. In a view where the burn out is certainly a problem of the whole healthcare organisation and different levels of interventions would be desirable, training self-awareness of workers in regard to their reactions to NICU environment also from the pre-employment stage could be an additional approach to prevent and manage it. More quality research would be advisable not only to identify the efficacy of interventions on individual well-being but also to test their long-term impact on productivity and cost-effectiveness.

Contributors IB and PDB conceived the study. IB and LF designed the search strategy and selection criteria. IB and LF reviewed all titles and abstracts for potentially eligible articles, and all studies meeting the inclusion criteria. IB and LF extracted relevant data according to prespecified criteria. IB wrote the initial paper. LF and PDB reviewed the paper. All authors approved the study as submitted.

Funding The authors have not declared a specific grant for this research from any funding agency in the public, commercial or not-for-profit sectors.

Competing interests None declared.

Patient consent for publication Not required.

Ethics approval Not required.

Provenance and peer review Not commissioned; externally peer reviewed.

ORCID iD

Ilia Bresesti http://orcid.org/0000-0002-6812-7556

\section{REFERENCES}

1 Maslach C, Schaufeli WB, Leiter MP. Job burnout. Annu Rev Psychol 2001:52:397-422

2 Shanafelt TD, West CP, Sinsky C, et al. Changes in burnout and satisfaction with worklife integration in physicians and the general us working population between 2011 and 2017. Mayo Clin Proc 2019;94:1681-94.

3 Shanafelt TD, Balch CM, Bechamps G, et al. Burnout and medical errors among American surgeons. Ann Surg 2010;251:995-1000.

4 Anagnostopoulos F, Liolios E, Persefonis G, et al. Physician burnout and patient satisfaction with consultation in primary health care settings: evidence of relationships from a one-with-many design. J Clin Psychol Med Settings 2012;19:401-10.

5 Dewa CS, Loong D, Bonato $S$, et al. How does burnout affect physician productivity? A systematic literature review. BMC Health Serv Res 2014;14:325.

6 Panagioti M, Geraghty K, Johnson J, et al. Association between physician burnout and patient safety, professionalism, and patient satisfaction: a systematic review and meta-analysis. JAMA Intern Med 2018;178:1317-30.

7 Tawfik DS, Profit J, Morgenthaler TI, et al. Physician burnout, well-being, and work unit safety grades in relationship to reported medical errors. Mayo Clin Proc 2018;93:1571-80

8 Shanafelt TD, Mungo M, Schmitgen J, et al. Longitudinal study evaluating the association between physician burnout and changes in professional work effort. Mayo Clin Proc 2016;91:422-31.
9 Braithwaite M. Nurse burnout and stress in the NICU. Adv Neonatal Care 2008;8:343-7.

10 Bellieni CV, Righetti P, Ciampa R, et al. Assessing burnout among neonatologists. J Matern Fetal Neonatal Med 2012:25:2130-4.

11 Profit J, Sharek PJ, Amspoker AB, et al. Burnout in the NICU setting and its relation to safety culture. BMJ Qual Saf 2014;23:806-13.

12 Prentice TM, Gillam L, Davis PG, et al. Always a burden? healthcare providers' perspectives on moral distress. Arch Dis Child Fetal Neonatal Ed 2018;103:F441-5.

13 Liberati A, Altman DG, Tetzlaff J, et al. The PRISMA statement for reporting systematic reviews and meta-analyses of studies that evaluate healthcare interventions: explanation and elaboration. BMJ 2009;339:b2700.

14 Zingg W, Castro-Sanchez E, Secci FV, et al. Innovative tools for quality assessment: integrated quality criteria for review of multiple study designs (ICROMS). Public Health 2016;133:19-37.

15 Weiner MF, Caldwell T, Tyson J. Stresses and coping in ICU nursing: why support groups fail. Gen Hosp Psychiatry 1983;5:179-83.

16 Rosenthal SL, Schmid KD, Black MM. Stress and coping in a NICU. Res Nurs Health 1989; 12:257-65.

17 Ewing AE, Carter BS. Once again, Vanderbilt NICU in Nashville leads the way in nurses' emotional support. Pediatr Nurs 2004;30:471-2.

18 Boos VD, Okah FA, Swinton CH, et al. The comprehensive care rounds: facilitating multidisciplinary communication among caregivers of complex patients in the neonatal intensive care unit. Adv Neonatal Care 2010;10:301-6.

19 O'Mahony S, Gerhart J, Abrams I, et al. A multimodal mindfulness training to address mental health symptoms in providers who care for and interact with children in relation to end-of-life care. Am J Hosp Palliat Care 2017:34:838-43.

20 Rippstein-Leuenberger K, Mauthner O, Bryan Sexton J, et al. A qualitative analysis of the three good things intervention in healthcare workers. BMJ Open 2017;7:e015826

21 D'Urso A, O'Curry S, Mitchell L, et al. Staff matter too: pilot staff support intervention to reduce stress and burn-out on a neonatal intensive care unit. Arch Dis Child Fetal Neonatal Ed 2019;104:F341.

22 Lary A, Borimnejad L, Mardani-Hamooleh M. The impact of a stress management program on the stress response of nurses in neonatal intensive care units: a quasiexperimental study. J Perinat Neonatal Nurs 2019;33:189-95.

23 Regehr C, Glancy D, Pitts A, et al. Interventions to reduce the consequences of stress in physicians: a review and meta-analysis. J Nerv Ment Dis 2014;202:353-9.

24 West CP, Dyrbye LN, Erwin PJ, et al. Interventions to prevent and reduce physician burnout: a systematic review and meta-analysis. Lancet 2016;388:2272-81.

25 Clough BA, March S, Chan RJ, et al. Psychosocial interventions for managing occupational stress and burnout among medical doctors: a systematic review. Syst Rev 2017:6:144

26 Panagioti M, Panagopoulou E, Bower $\mathrm{P}$, et al. Controlled interventions to reduce burnout in physicians: a systematic review and meta-analysis. JAMA Intern Med 2017:177:195-205.

27 Romppanen J, Häggman-Laitila A. Interventions for nurses' well-being at work: a quantitative systematic review. J Adv Nurs 2017;73:1555-69.

28 Irving JA, Dobkin PL, Park J. Cultivating mindfulness in health care professionals: a review of empirical studies of mindfulness-based stress reduction (MBSR). Complement Ther Clin Pract 2009;15:61-6.

29 Krasner MS, Epstein RM, Beckman $\mathrm{H}$, et al. Association of an educational program in mindful communication with burnout, empathy, and attitudes among primary care physicians. JAMA 2009;302:1284-93

30 Bazarko D, Cate RA, Azocar F, et al. The impact of an innovative Mindfulness-Based stress reduction program on the health and well-being of nurses employed in a corporate setting. J Workplace Behav Health 2013;28:107-33.

31 Fortney L, Luchterhand C, Zakletskaia L, et al. Abbreviated mindfulness intervention for job satisfaction, quality of life, and compassion in primary care clinicians: a pilot study. Ann Fam Med 2013;11:412-20.

32 Kulka JM, De Gagne JC, Mullen CK, et al. Mindfulness-Based stress reduction for newly graduated registered nurses. Creat Nurs 2018;24:243-50.

33 Okah FA, Wolff DM, Boos VD, et al. Perceptions of a strategy to prevent and relieve care provider distress in the neonatal intensive care unit. Am J Perinatol 2012:29:687-92. 